\title{
Morbid Parasitological and Histopathological Events in Hamsters Infected with Intestinal Amoebiasis Given Artesunate
}

\author{
Soheir Mahmoud and Nevine Guirguis Nessim \\ Department of Parasitology, Theodor Bilharz Research Institute, Imbaba, Giza 12411, Egypt \\ Correspondence should be addressed to Nevine Guirguis Nessim, habib.elmasry@gmail.com
}

Received 22 April 2008; Accepted 16 December 2008

Objectives. This work is a trial to elucidate the parasitological and histopathological sequelae of giving the antimalarial drug (artesunate) in experimental intestinal amoebiasis. Methods. A group of 24 hamsters was infected by Entamoeba histolytica cysts orally using a canula. This group was subdivided into two main subgroups. Subgroup I: given 6000 E. histolytica cysts orally by a canula, then sacrificed five weeks postinfection. Subgroup II : given the same infective dose, then two weeks later, treated with artesunate $10 \mathrm{mgm} / \mathrm{Kg}$ b. wt/hamster over 3 consecutive days. Again sacrifice was performed five weeks postinfection. Multiple stool examinations, and histopathological examination of the caecal end of the large intestine were resorted to, in order to assess the antiamoebic effect of the drug. Faecal smear examination revealed absolute disappearance of E. histolytica cysts in the treated group. Again, histopathology of the mucosal scrapings of the caecum showed complete absence of Entamoeba histolytica trophozoites in the treated group, when compared to the control animals $(P<.001)$. This study may be beneficial, especially in areas endemic with amoebiasis to help overcoming the emerging resistance to the usually available antiamoebic drugs.

Copyright (C) 2008 S. Mahmoud and N. Guirguis Nessim. This is an open access article distributed under the Creative Commons Attribution License, which permits unrestricted use, distribution, and reproduction in any medium, provided the original work is properly cited.

\section{Introduction}

Fernández et al. [1] postulated that the aqueous, methanol, acetone, and hexane leaf extracts from mature Artemisia plants were found to be active in vitro against the parasitic protozoa Entamoeba histolytica and Giardia lamblia.

Amoebiasis is a protozoal disease which is widespread primarily in developping countries. Infections are correlated with poor hygienic conditions, poor water quality control, and overcrowding. Other risk factors include young age, poor personnal hygiene, risky social behaviour, malnutition, and hypochlorhydria [2].

The goldstandard of this study is to evaluate both the parasitological and the pathological effects of the antimalarial drug, artesunate in experimental intestinal amoebiasis.

\section{Material and Methods}

A group of fifteen golden Syrian hamsters (weighing $100 \mathrm{gm}$ each) was used in the experiment. This group was further subdivided into two small subgroups. Subgroup I: constituted control infected untreated hamsters. Infection was done by oral administration of 6000 Entamoeba histolytica cysts through an oesophageal tube. Subgroup II: constituted infected animals, treated three weeks later with three consecutive oral doses of $10 \mathrm{mg}$ artesunate $/ \mathrm{Kg} \mathrm{b}$. wt over three successive days. Animals were kept on a standard diet, under $24^{\circ} \mathrm{C}$ for three weeks in the Biological Unit at TBRI. Treatment was initiated three weeks postinfection. The drug was supplied by Theodor Bilharz Research Institute, Giza, Egypt. The manufacturer is Erfa. The experiment was carried according to the internationally valid guidelines in the Theodor Biharz Research institute. All animals were sacrificed five weeks postinfection and two weeks posttreatment.

\subsection{Stool Parasitology}

2.1.1. Direct Smear Method. A stool sample from each infected hamster was examined by direct smear method in order to confirm the infection [3]. A wet mount with saline and Lugol's iodine (Dobell reactive) was prepared, and microscopic examination for motile trophozoites and Entamoeba cysts was carried out using the low and high power $(\times 200$ and $\times 400)$. 
2.1.2. Concentration Method MIFC. Faecal samples were collected from hamsters in clean small Petri dishes. The samples were microscopically examined by merthiolate iodine formaldehyde concentration (MIFC) method [4]. From each sample, about one gram was taken and emulsified in a tube containing five $\mathrm{mL}$ of merthiolate iodine formaldehyde (MIF) solution. The mixture was strained through household tea sieves into a $15-\mathrm{mL}$ centrifuge tube. Seven $\mathrm{mL}$ of cold ether (kept in refrigerator at $4^{\circ} \mathrm{C}$ ) were added to the top centrifuge tube. Stopper was inserted, and the tube was shacked vigorously (in case that ether remains on top after shacking, $1 \mathrm{~mL}$ tap water was added and the tube shacked again). The stopper was removed, and the tube was allowed to stand for 2 minutes. The tube was centrifuged for 5 minutes at $3500 \mathrm{~g}$ (gravity). After centrifugation, 4 layers appeared in the tube: an ether layer on top, a plug of fecal detritus, an MIF layer, then the sediment, which contains Entamoeba cysts. All layers were poured off except the bottom layer of the sediment. The sediment was mixed; a drop was put on a slide, covered and examined microscopically.

2.2. Histopathological Studies. After sacrifice of the animals, sections from the caecal end of the large intestine were taken, embedded in paraffin, and sectioned. Five sections (5 microns in thickness) were taken from each large intestine specimen, each section being at a distance of at least $500 \mu \mathrm{m}$ from the preceding one. Sections were stained with haematoxylin and eosin [5]. In addition, examination of inflammatory infiltrate particularly of plasmacellular type was done.

\section{Statistics}

Comparison was done between each treated group and its respective untreated control. The percentage change between each two groups to be compared was assessed using the formula

((Mean value of the first group

- mean value of the second group) $\times 100$ )

$\times(\text { Mean value of the first group })^{-1}$.

Differences between the mean scores of any of the two groups to be compared were tested for significance, using an unpaired 2-tailed Student's $t$-test. The data were considered significant if $P$ values were less $<.05$.

\section{Results}

4.1. Parasitological Parameter (Faecal Smear Examination). The mean parasitic count by field microscopy in the control and treated groups, groups I and II, as shown in Table 1 was ( $40 \pm 7.2$, and $2.4 \pm 1.00$, resp.). The difference between the treated groups was statistically significant from respective untreated control hamsters at $P<.001$. Again, mucosal scrapings of the caecum revealed $1.8+0.6$ Entamoeba trophozoites in the treated group compared to $18.4+6.8$ in
TABLE 1: Faecal trophozoite and/or cyst count, and caecal Mucosal scrapings of infected hamsters given 6000 E. histolytica cysts orally, treated 3 weeks later with artesunate $10 \mathrm{mgm} / \mathrm{Kg}$ b. wt, sacrificed 2 weeks later ( 5 weeks postinfection).

\begin{tabular}{lll}
\hline \multirow{3}{*}{ Animal group } & $\begin{array}{l}\text { Number of Entamoeba } \\
\text { histolytica trophozoites } \\
\text { and/or cysts/gm. faeces }\end{array}$ & $\begin{array}{l}\text { Number of Entamoeba } \\
\text { histolytica trophozoites } \\
\text { and/or cysts in the cae- }\end{array}$ \\
$\left(10^{6}\right)$ & cum/HP field \\
\hline
\end{tabular}

Group I: control

infected untreated

$40 \pm 7.2$

$18.4 \pm 6.8$

hamsters

Group II: treated

with $10 \mathrm{mmg} / \mathrm{Kg}$

b. wt artesunate

triple divided do-

$2.4+1.00^{* * *}$

$1.8+0.6^{* * *}$

se on three succe-

ssive days

*** Means statistically significant difference from infected control untreated hamsters at $P<.001$.

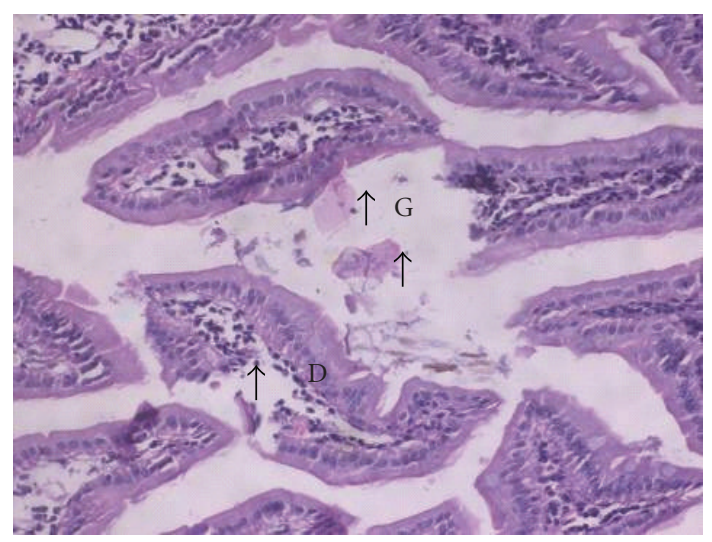

Figure 1: Control infected Giardia. Degeneration and eruption of intestinal villi in infected hamster (H\&E 400x). G = Giardia; D = degenerated endothelial cells.

the control untreated group. The difference was statistically significant at $P<.001$ (see Table 1 ).

4.2. Pathological Parameter (Caecal Mucosal Scrapings). In the control infected nontreated hamsters, atrophic degeneration of the intestinal villi was shown (see Figure 1). While the treated group revealed complete regeneration of the endothelial cells as shown by haematoxylin and eosin stain (see Figure 2 and 3).

\section{Discussion}

Amoebiasisis a common parasitic event with worldwide distribution. It can usually spread by direct faeco-oral transmission.

Long before, Borst and Ouellette [6] stated that although studies of resistance mechanisms in parasites have lagged behind similar studies in bacteria and cancer cells, the tools to tackle this problem are rapidly improving. Transformation 


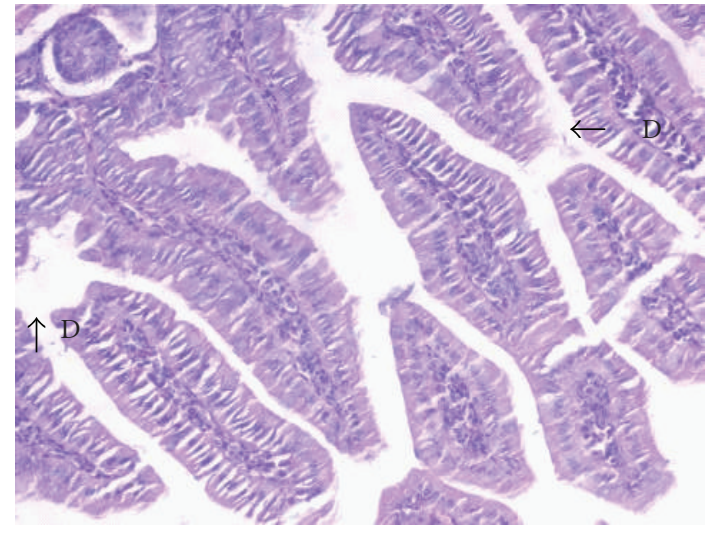

FIGURE 2: Intestinal villi in single treated infected hamster showing partial regeneration but still degenerated endothelial cells could be detected (H\&E 400x). G = Giardia; $\mathrm{D}=$ degenerated endothelial cells.

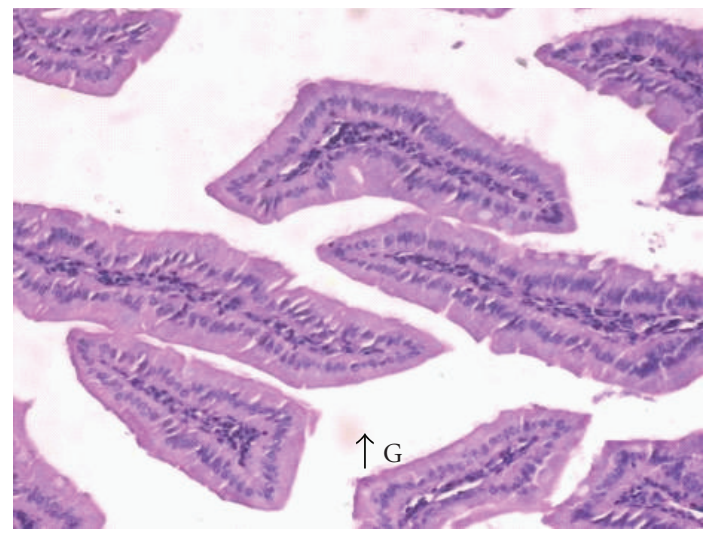

FIGURE 3: Intestinal villi in double treated infected hamster showing completed regeneration of endothelial cells ( $\mathrm{H} \& \mathrm{E} 400 \mathrm{x})$. $\mathrm{G}=$ Giardia; D = degenerated endothelial cells.

with exogenous DNA is now possible with all major parasitic protozoa of humans. Hence, putative resistance genes can be tested in sensitive protozoa, allowing an unambiguous reconstruction of resistance mechanisms. Again, the authors approved that gene cloning, the polymerase chain reaction, and monoclonal antibodies against resistance-related proteins have made it possible to analyse potential resistance mechanisms in the few parasites that can be obtained from infected people. Loss of drug activation is the main mechanism of metronidazole resistance in Trichomonas and Giardia species. Finally, the authors concluded that a decrease of the proximal cellular electron donor for metronidazole activation, ferrodoxin, is the main cause of resistance in Trichomonas [6].

The goldstandard in the defense mechanisms now available against parasitic protozoa is chemotherapy. This defense is being eroded by drug resistance, and with few new drugs in the pipeline, prevention and circumvention of resistance are medical and veterinary priorities. By the advent of many antiamoebic preparations, and by the emergence of drug resistance to some of them, this experiment has been resorted to solve the problem of drug resistance to Entamoeba histolytica infection. From this study, it could be concluded that the antimalarial drug artesunate exerts a potent antiamoebic effect in experimental animals. This is evident by the absence of faecal cysts recovered in the treated group. Again, this drug regimen was shown to ameliorate the deleterious effect of Entamoeba histolytica upon the caecal mucosa. This is evidenced by complete villus regeneration recovered in this previously mentionned group. Previously, Fernández et al. [1] noted that the inhabitants of Northeast of Mexico use an infusion of leaves from Artemisia ludoviciana as an antidiarrheal remedy. The authors postulated that the aqueous, methanol, acetone, and hexane leaf extracts from mature plants were found to be active in vitro against the parasitic protozoa Entamoeba histolytica and Giardia lamblia.

\section{Conclusion}

The aim of this study was to testify the noxious imprint of the antimalarial drug artesunate, in intestinal amoebiasis. As a worldwide concern, this could be of crucial importance. In endemic areas like Egypt, amoebaisis is a common parasitic event, and resistance to the commonly used antiamoebic drugs constitutes a major health problem.

\section{Recommendations}

Further trials are being recommended to discover new potent antiamoebic compounds. The experiments conducted for this paper are according to the law and regulations of Egypt as well as the scientific ethics of the profession.

\section{References}

[1] S. S. Fernández, M. C. R. Guerra, B. D. M. Cárdenas, J. V. Villarreal, and L. V. Treviño, "In vitro antiprotozoal activity of the leaves of Artemisia ludoviciana," Fitoterapia, vol. 76, no. 5, pp. 466-468, 2005.

[2] A. Pietrzak, G. Chodorowska, J. Urban, V. Bogucka, and E. Dybiec, "Cutaneous manifestation of giardiasis-case report," Annals of Agricultural and Environmental Medicine, vol. 12, no. 2, pp. 299-303, 2005.

[3] M. Cheesbrough, Medical Laboratory Manual for Tropical Countries. Vol. 1, ELBS, Tropical Health Technology, Butterworths, Cambridge, UK, 2nd edition, 1987.

[4] W. Blagg, E. L. Schloegel, N. S. Mansour, and G. I. Khalaf, "A new concentration technique for the demonstration of protozoa and helminth eggs in feces," American Journal of Tropical Medicine and Hygiene, vol. 4, pp. 23-28, 1955.

[5] F. C. von Lichtenberg, "Host response to eggs of Schistosoma mansoni. I. Granuloma formation in the unsensitized laboratory mouse," American Journal of Pathology, vol. 41, pp. 711$731,1962$.

[6] P. Borst and M. Ouellette, "New mechanisms of drug resistance in parasitic protozoa," Annual Review of Microbiology, vol. 49, pp. 427-460, 1995. 